\title{
Non-culprit lesion percutaneous coronary intervention during acute myocardial infarction - the road not taken?
}

\author{
Guy Witberg', Ran Kornowski \\ ${ }^{1}$ Department of Cardiology, Rabin Medical Center, Petach Tikva, Israel \\ ${ }^{2}$ Sackler School of Medicine, Tel Aviv University, Tel Aviv, Israel
}

Postep Kardiol Inter 2015; 11, 2 (40): 71-73 DOI: $10.5114 /$ pwki.2015.52277

In this issue of "Postępy w Kardiologii Interwencyjnej/Advances in Interventional Cardiology" journal, 1 case report and 1 original article present one of the most hotly debated dilemmas in the current practice of interventional cardiology - the optimal revascularization approach for patients undergoing primary percutaneous coronary interventions (PPCI) for myocardial infarction (MI) and found to have multivessel coronary artery disease (CAD).

The case report by Wolny et al. describes the clinical course of a patient who was admitted directly to the catheterization laboratory due to an inferior wall ST elevation MI (STEMI), as a first presentation of CAD. On angiography, the patient was found to have a two-vessel CAD - an occlusive lesion in the distal right coronary artery (RCA), the culprit lesion in the infarct-related artery (IRA) this context, and a second lesion in the proximal left anterior descending artery (LAD) with involvement of the $1^{\text {st }}$ diagonal branch (the non-IRA lesion) without obstruction of the coronary flow. The patient underwent successful PPCI of the culprit lesion, with resolution of symptoms, and was admitted for continued care, but a few hours later developed anterior $\mathrm{MI}$ due to thrombotic occlusion of the LAD stenosis, not treated during the PPCI.

This case raises the obvious question - could the $2^{\text {nd }}$ $\mathrm{MI}$ have been avoided by preventive stenting of the nonIRA lesion during the PPCI? The answer to this question is not clear. Currently, such patients present major dilemmas for the interventional cardiologist: patients with multivessel CAD comprise over half of the STEMI population, and their prognosis is worse, compared to patients with single vessel disease [1]. Considering the well-established prognostic benefit from achieving complete revascularization (or at least "reasonable" incomplete revascularization) [2, 3] in CAD patients, it is obvious that the optimal goal is a more aggressive approach leading to more complete revascularization. The question at hand is the optimal timing of revascularization for non-IRA lesions.

Current guidelines [4] based upon a firm base of evidence from large observational studies (in a field that until recently was seriously lacking in randomized trials) [5], which found a significant increase in adverse outcomes for patients undergoing multivessel $\mathrm{PCl}$ in the setting of acute $\mathrm{MI}$, recommend a restrictive approach that discourages treatment of non-IRA lesions during the index $\mathrm{PCl}$, unless the patient is in cardiogenic shock. This dogma has been challenged in recent years by evidence from randomized trials [6, 7], which found significant reductions in composite ischemic endpoints, when performing "preventive" multivessel revascularization during STEMI.

In spite of the impressive results from these trials, it should be remembered that the PRAMI trial [6] has been criticized for having been stopped prematurely due to a much higher than expected treatment effect and the achieved statistically significant results with a relatively small number of clinical events raises concerns of a chance finding amplified due to the early termination of the trial. The CVLPRIT trial [7] was not able to show reductions in "hard" endpoints, and the benefit in the composite endpoint was solely driven by repeat revascularizations. Perhaps the most interesting and relevant trial in this field to date is the PRIMULTI trial [8], recently presented at the American College of Cardiology annual conference, which represents a middle ground between the guideline-recommended culprit lesion only approach and the en vogue preventive $\mathrm{PCl}$ strategy - early (i.e., within the same hospitalization) staged $\mathrm{PCl}$, an approach whose advantages over preventive $\mathrm{PCl}$ have previously been suggested by a post hoc analysis of the

Corresponding author:

Prof. Ran Kornowski, Department of Cardiology, Rabin Medical Center, 100 Jabutinski St, 49100 Petach Tikva, Israel, phone: 972 39377107, e-mail: ran.kornowski@gmail.com

Received: 29.03.2015, accepted: 29.03.2015. 
HORIZONS-AMI trial [9], in this case (the PRIMULTI trial) integrated with fractional flow reserve (FFR) evaluation of the non-IRA lesions.

The drawbacks of multivessel interventions during $\mathrm{PPCl}$ - most notably prolonging procedural time and contrast exposure, putting the patient at increased risk for procedure-related complications, and overestimation of the severity of non-IRA lesions, leading to unnecessary stenting - should not be forgotten. And perhaps an interim approach, as shown in the PRIMULTI trial, combines the best of all worlds - leaving the patient with as complete revascularization as possible, while avoiding the hazards of multiple interventions in the acute setting.

As demonstrated very vividly by the case report at hand, each choice has its drawbacks. Hopefully, more definite answers to the dilemmas presented by this case will be given by larger RCTs due to be published during the coming years, most notably the COMPLETE (NCT01740479) and COMPARE-ACUTE (NCT01399736) trials. Until the results of such trials are revealed, caution needs to be exercised and the risk benefit profile for the individual patient contemplated prior to performing multivessel interventions in the setting of STEMI.

The article by Siudak et al. represents a commendable attempt to gain insight into a related, although far less prevalent dilemma - how to treat multiple lesions within the IRA. Their registry (CORAMI) is the first attempt to prospectively evaluate the different approaches to these lesions (i.e. complete IRA revascularization vs. culprit lesion only revascularization), and although the authors had to change the design of the trial from a multicenter randomized trial to a registry due to a slow recruitment rate, their results are interesting and warrant attention: a consistent trend in favor of the culprit lesion only approach was found for both in-hospital (mortality, stent thrombosis, angiographic complications and urgent repeat revascularizations), and 12-month (mortality, stent thrombosis, $\mathrm{Ml}$ and urgent repeat revascularizations) outcomes. Although the results did not reach statistical significance due to the low sample size, the absolute margin in outcomes is quite impressive and definitely clinically significant. The most plausible explanation for these findings, suggested by the predominance of stent thrombosis among the clinical outcomes, is stenting of non-significant lesions, due to overestimation of their severity during the acute $\mathrm{Ml}$ phase, a consequence that may have been avoided by using a more methodical process for the evaluation of non-culprit lesions, such as FFR or intravascular ultrasound (IVUS). Another important lesson to be learned from the results of the CORAMI registry is the increased risk for procedural complications when performing multiple interventions during acute $\mathrm{MI}$ - as is evident from the almost doubling of the rate of angiographic complications, a lesson that definitely extends to treatment of non-IRA lesions as well.
The results of the CORAMI registry reported by Siudak et al. should be viewed in two perspectives:

1. As a hypothesis generating data for the planning of future trials investigating the issue of treating multiple lesions within an IRA in the setting of MI.

2. As another reminder of the hazards of multiple coronary interventions in the acute $\mathrm{PPCl}$ setting, an issue more important to remember considering the recent trend towards a more aggressive and complete revascularization approach in patients with multivessel STEMI.

Hopefully, this project will be ongoing and supply us with future data from a larger sample size to gain more knowledge as to the risks and benefits of multiple coronary interventions during acute $\mathrm{MI}$, to help clinicians make informed and evidence-based choices as to the optimal treatment for these high-risk patients.

Two roads diverged in a yellow wood,

And sorry I could not travel both

And be one traveler, long I stood

And looked down one as far as I could

To where it bent in the undergrowth.

Then took the other, as just as fair, And having perhaps the better claim, Because it was grassy and wanted wear; Though as for that the passing there Had worn them really about the same,

And both that morning equally lay In leaves no step had trodden black. Oh, I kept the first for another day! Yet knowing how way leads on to way, I doubted if I should ever come back.

I shall be telling this with a sigh Somewhere ages and ages hence: Two roads diverged in a wood, and I I took the one less traveled by, And that has made all the difference.

Robert Frost - The Road Not Taken, Mountain Interval, 1916.

\section{Conflict of interest}

The authors declare no conflict of interest.

\section{References}

1. Park DW, Clare RM, Schulte PJ, et al. Extent, location, and clinical significance of non-infarct-related coronary artery disease among patients with ST-elevation myocardial infarction. JAMA 2014; 312: 2019-27.

2. Garcia S, Sandoval Y, Roukoz H, et al. Outcomes after complete versus incomplete revascularization of patients with multivessel coronary artery disease: a meta-analysis of 89,883 patients enrolled in randomized clinical trials and observational studies. J Am Coll Cardiol 2013; 62: 1421-31. 
3. Farooq V, Serruys PW, Bourantas CV, et al. Quantification of incomplete revascularization and its association with five-year mortality in the synergy between percutaneous coronary intervention with taxus and cardiac surgery (SYNTAX) trial validation of the residual SYNTAX score. Circulation 2013; 128: 141-51.

4. Windecker S, Kolh P, Alfonso F, et al. 2014 ESC/EACTS Guidelines on myocardial revascularization: The Task Force on Myocardial Revascularization of the European Society of Cardiology (ESC) and the European Association for Cardio-Thoracic Surgery (EACTS) Developed with the special contribution of the European Association of Percutaneous Cardiovascular Interventions (EAPCI). Eur Heart J 2014; 35: 2541-619.

5. Vlaar PJ, Mahmoud KD, Holmes DR Jr, et al. Culprit vessel only versus multivessel and staged percutaneous coronary intervention for multivessel disease in patients presenting with ST-segment elevation myocardial infarction: a pairwise and network meta-analysis. J Am Coll Cardiol 2011; 58: 692-703.

6. Wald DS, Morris JK, Wald NJ, et al. Randomized trial of preventive angioplasty in myocardial infarction. N Engl J Med 2013; 369: 1115-23.

7. Gershlick AH, Khan JN, Kelly DJ, et al. Randomized trial of complete versus lesion-only revascularization in patients undergoing primary percutaneous coronary intervention for STEMI and multivessel disease: the CvLPRIT Trial. J Am Coll Cardiol 2015; 65: 963-72.

8. Engstrøm T, The Third DANish Study of Optimal Acute Treatment of Patients with ST-segment Elevation Myocardial Infarction PRImary PCI in MULTIvessel Disease - DANAMI3-PRIMULTI. Presented at the $64^{\text {th }}$ annual scientific session of the American College of Cardiology. San Diego CA USA March $16^{\text {th }} 2015$.

9. Kornowski R, Mehran R, Dangas G, et al. Prognostic impact of staged versus "one-time" multivessel percutaneous intervention in acute myocardial infarction: analysis from the HORIZONS-AMI (harmonizing outcomes with revascularization and stents in acute myocardial infarction) trial. J Am Coll Cardiol 2011; 58: 704-11. 